Western University

Scholarship@Western

7-6-2017

\title{
Maternal nicotine exposure leads to decreased cardiac protein disulfide isomerase and impaired mitochondrial function in male rat offspring.
}

Nicole G Barra

Maria Lisyansky

Taylor A Vanduzer

Sandeep Raha

Alison C Holloway

See next page for additional authors

Follow this and additional works at: https://ir.lib.uwo.ca/physpharmpub

Part of the Medical Physiology Commons, Pharmacy and Pharmaceutical Sciences Commons, and the Reproductive and Urinary Physiology Commons

\section{Citation of this paper:}

Barra, Nicole G; Lisyansky, Maria; Vanduzer, Taylor A; Raha, Sandeep; Holloway, Alison C; and Hardy, Daniel $B$, "Maternal nicotine exposure leads to decreased cardiac protein disulfide isomerase and impaired mitochondrial function in male rat offspring." (2017). Physiology and Pharmacology Publications. 97. https://ir.lib.uwo.ca/physpharmpub/97 


\section{Authors}

Nicole G Barra, Maria Lisyansky, Taylor A Vanduzer, Sandeep Raha, Alison C Holloway, and Daniel B Hardy

This article is available at Scholarship@Western: https://ir.lib.uwo.ca/physpharmpub/97 


\title{
Maternal nicotine exposure leads to decreased cardiac protein disulfide isomerase and impaired mitochondrial function in male rat offspring
}

\author{
Nicole G. Barra ${ }^{1 *}$ | Maria Lisyansky ${ }^{1 *}$ | Taylor A. Vanduzer ${ }^{2}$ | Sandeep Raha ${ }^{3}$ | \\ Alison C. Holloway ${ }^{2}$ (D) । Daniel B. Hardy ${ }^{1,4}$
}

${ }^{1}$ Department of Physiology and

Pharmacology, Western University, London, Ontario, Canada

${ }^{2}$ Department of Obstetrics and Gynecology, McMaster University, Hamilton, Ontario,

Canada

${ }^{3}$ Department of Pediatrics, McMaster University, Hamilton, Ontario, Canada

${ }^{4}$ Departments of Obstetrics and Gynecology, Children's Health Research Institute, Lawson, Health Research Institute, Western University, London, Ontario, Canada

Correspondence

Daniel B. Hardy, Department of Physiology and Pharmacology, University of Western Ontario, London, Ontario, Canada N6A 5C1 Email: daniel.hardy@schulich.uwo.ca

Funding information

Whaley Fellowship; Women's Development

Council; Dean's Undergraduate Research

Opportunities Program award at Western University; Molly Towell Perinatal Research Foundation; Canadian Institutes of Health Research, Grant/Award Number: MOP 111011 and MOP86474

\begin{abstract}
Smoking throughout pregnancy can lead to complications during gestation, parturition and neonatal development. Thus, nicotine replacement therapies are a popular alternative thought to be safer than cigarettes. However, recent studies in rodents suggest that fetal and neonatal nicotine exposure alone results in cardiac dysfunction and high blood pressure. While it is well known that perinatal nicotine exposure causes increased congenital abnormalities, the mechanisms underlying longer-term deficits in cardiac function are not completely understood. Recently, our laboratory demonstrated that nicotine impairs placental protein disulfide isomerase (PDI) triggering an increase in endoplasmic reticulum stress, leading us to hypothesize that this may also occur in the heart. At 3 months of age, nicotine-exposed offspring had $45 \%$ decreased PDI levels in the absence of endoplasmic reticulum stress. Given the association of PDI and superoxide dismutase enzymes, we further observed that antioxidant superoxide dismutase-2 levels were reduced by $32 \%$ in these offspring concomitant with a $26-49 \%$ decrease in mitochondrial complex proteins (I, II, IV and V) and tissue inhibitor of metalloproteinase-4, a critical matrix metalloprotease for cardiac contractility and health. Collectively, this study suggests that perinatal nicotine exposure decreases PDI, which can promote oxidative damage and mitochondrial damage, associated with a premature decline in cardiac function.
\end{abstract}

\section{KEYWORDS}

heart, mitochondria, perinatal nicotine exposure, protein disulfide isomerase

\section{1 | INTRODUCTION}

Despite increased knowledge and education regarding the health risks of smoking, it is surprising that approximately $20 \%$ of Canadian women still smoke during pregnancy, with the highest prevalence in the Northern Territories at 59\% (Cui, Shooshtari, Forget, Clara, \& Cheung, 2014). Owing to the highly addictive nature of nicotine in cigarettes, many pregnant women turn to nicotine replacement therapies as a method of smoking cessation. These therapies include such products as nicotine gum, lozenges, nasal spray and the nicotine patch. While they are thought to be safer than conventional cigarettes, the long-term health effects of early life exposure to nicotine remain elusive (De Long, Barra, Hardy, \& Holloway, 2014). Regardless

*Both authors contributed equally to this manuscript. of its source, animal models have revealed that nicotine use during pregnancy leads to adverse metabolic, neurobehavioral, fertility, pulmonary and cardiovascular outcomes in the offspring (Chou \& Chen, 2014; Dasgupta, Xiao, Xu, Yang, \& Zhang, 2012; Gao et al., 2005, 2008; Holloway, Kellenberger, \& Petrik, 2006; Ma, Nicholson, Wong, Holloway, \& Hardy, 2014; Pauly \& Slotkin, 2008; Wang et al., 2015; Yu et al., 2016).

With respect to the postnatal cardiovascular health of the offspring, perinatal nicotine exposure results in a number of adverse cardiac outcomes including increased heart rate and blood pressure, thicker septum, decreased ejection volume, and a reduced ability to recover from ischemic insults (Fox et al., 2012; Gao et al., 2008; Lawrence et al., 2008; Xiao et al., 2016; Yu et al., 2016). Moreover, these offspring exhibit ventricular-arterial stiffening, underdeveloped elastic fibers and impaired acetylcholine-induced aortic relaxation, with a 
greater incidence in males (Chou \& Chen, 2014; Fox et al., 2012; Gao et al., 2008; Lawrence et al., 2008; Wang et al., 2015; Xiao et al., 2008; Yu et al., 2016). Elegant studies by Chou and Chen have demonstrated some of these heart deficits are due, in part, to nicotine-induced cardiac remodeling (i.e. increased collagen formation, cardiomyocyte width and higher $\beta$-myosin heavy chain expression) by 3 weeks of age (Chou \& Chen, 2014). By 3 months of age, this collagen formation continues leading to myocardial fibrosis (Yu et al., 2016). Yet to date, little is known about the molecular mechanisms underlying the long-term cardiac dysfunction and fibrosis observed. Therefore, an initial toxicological investigation to determine mechanisms associated with cardiac dysfunction is warranted. Recently we have found that maternal nicotine exposure (MNE) in vivo leads to decreased protein disulfide isomerase (PDI) in rat placenta associated with endoplasmic reticulum (ER) stress and placental insufficiency (Holloway et al., 2014; Wong, Nicholson, Holloway, \& Hardy, 2015). As PDI has been implicated in cardioprotection and as a survival factor during ischemia, it is conceivable that cardiac PDI could be altered in these MNE offspring, which exhibit impaired recovery to ischemic injury (Lawrence et al., 2008; Toldo, Boccellino, et al. 2011a; Toldo, Severino, Abbate, \& Baldi, 2011b; Xiao et al., 2016). Moreover, given a decrease in PDI leads to ER and oxidative stress, which can also impede cardiac function, it is very likely these cell stresses are also aggravated in the MNE heart (Rush, Green, Maclean, \& Code, 2005; Yu et al., 2012). Therefore, the aim of this study was to determine whether nicotine exposure in vivo leads to impaired cardiac PDI levels in early and late postnatal life. If so, we then wanted to uncover the downstream mechanisms contributing to the development of cardiac dysfunction.

\section{2 | MATERIALS AND METHODS}

\section{1 | Maternal nicotine exposure rat model}

Animal experiments were approved by the Animal Research Ethics Board at McMaster University, in accordance with the guidelines of the Canadian Council for Animal Care. All rats were conventionally housed in polycarbonate microisolator cages under controlled lighting (12: 12 light/dark), humidity $(40-50 \%)$ and temperature $\left(22^{\circ} \mathrm{C}\right)$ with ad libitum access to water and standard chow diet (Teklad 22/5 rodent diet; Envigo, Indianapolis, IN). Nulliparous 200-250 g female Wistar rats (Harlan, Indianapolis, IN, USA) were randomly assigned to receive daily subcutaneous injections of saline (vehicle) or nicotine bitartrate ( $1 \mathrm{mg} \mathrm{kg}^{-1} \mathrm{day}^{-1}$; Sigma-Aldrich, St. Louis, MO, USA) 2 weeks before mating, during gestation and until weaning (postnatal day 21). This dose of nicotine was previously shown to result in maternal serum cotinine, the major metabolite of nicotine, concentrations of $135.9 \pm 7.86 \mathrm{ng} \mathrm{ml}^{-1}$, which is comparable to concentrations in the serum of "moderate" female smokers or nicotine replacement therapy users (80 $\mathrm{ng} \mathrm{ml}^{-1}$ ) (Holloway et al., 2006). Litters were culled to eight at birth but only male offspring were used in this study to prevent confounding effects caused by the female reproductive cycle. Following weaning, rats were housed as sibling pairs until 6 weeks of age when they were individually housed. At 3 weeks (weaning) and
3 months of age, male offspring were killed via carbon dioxide inhalation. Hearts were extracted, frozen in liquid nitrogen and stored at $-80^{\circ} \mathrm{C}$ for analysis. Three-week offspring were chosen as this is the longest period of direct nicotine exposure while 3-month offspring were analyzed since this was earliest time point post-weaning where evidence of cardiac dysfunction has been reported (Chou \& Chen, 2014; Yu et al., 2016).

\section{2 | Protein extraction and Western blot}

Whole hearts were homogenized in RIPA buffer $(50 \mathrm{mM}$ Tris- $\mathrm{HCl}$, $\mathrm{pH}$ 7.4, $150 \mathrm{mM} \mathrm{NaCl}, 1 \mathrm{mM}$ EDTA, 1\% Nonidet P40, 0.25\% $\mathrm{C}_{24} \mathrm{H}_{39} \mathrm{NaO}_{4}$, supplemented with phosphatase inhibitors $(20 \mathrm{mM}$ $\mathrm{NaF}, 40 \mathrm{mM}$ sodium pyrophosphate, $40 \mathrm{mM} \mathrm{Na}_{3} \mathrm{VO}_{4}, 200 \mathrm{mM} \beta$-glycerophosphate disodium salt hydrate), and a protease inhibitor cocktail (Roche, Mississauga, ON). The solution was sonicated at 30\% amplitude for 5 seconds total, 1 second per pulse. It was then mixed in a rotator for 10 minutes at $4^{\circ} \mathrm{C}$ and centrifuged at $300 \mathrm{~g}$ for 15 minutes at $4^{\circ} \mathrm{C}$. The supernatant was collected and centrifuged at $16000 \mathrm{~g}$ for 20 minutes at $4^{\circ} \mathrm{C}$. The resulting supernatant was collected as the total cellular protein extract and quantified by colorimetric DC protein assay (Bio-Rad). Loading samples were prepared with fresh total cellular protein extract (avoiding repeated freeze-thaw cycles), NuPAGE LDS Sample Buffer (4x) (Invitrogen, Burlington, ON), NuPAGE Reducing Agent (10x) (Invitrogen) and deionized water, and then heated at $50^{\circ}$ $\mathrm{C}$ for 10 minutes to denature the proteins. Proteins (20 $\mu \mathrm{g}$ per well) were separated by size via gel electrophoresis in gradient polyacrylamide gels (Novex, Burlington, $\mathrm{ON}$ ) and transferred on to polyvinylidene difluoride membrane (Millipore, Etobicoke, Ontario). Membranes were blocked in 1× Tris-buffered saline-Tween 20 buffer with $5 \%$ non-fat milk (blocking solution) and then probed using the following primary antibodies: (i) KDEL/Grp78 (10C3; 1:300; sc-58774), CREB-2 (H-290; 1:300; sc-22800), Quiescin Q6 (QSOX1; G-12; 1:500; sc-160084), tissue inhibitor of metalloproteinase (TIMP) 4 (1:300; sc-9375), superoxide dismutase (SOD)-1 (1:1000; sc11407), SOD-2 (1:1000; sc-30080), heme oxygenase (HO)-2 (1:500; sc-11361; Biotechology, Santa Cruz, Dallas, Texas); (ii) phospho-PERK [Thr980] (16F8; 1:500; 3179), PERK (D11A8; 1:500; 5683), phospho-elF2a [Ser51] (119A11; 1:1000; 3597), elF2a (9722), PDI (C81H6; 1:1000; 3501), BiP (C50B12; 1:1000; 3177), GRP94 (1:1000; 2104; Cell Signaling, Danvers, MA); and (iii) OXPHOS (1:500; ab110413) (Abcam, Toronto, ON). Rabbit polyclonal citrate synthase primary antibody was generously donated by Dr. Sandeep Raha at McMaster University (Hamilton, ON, Canada) and diluted to $1: 1000$. Secondary donkey antibodies were used to detect the species-specific portion of the primary antibody, at the following dilutions: antirabbit 1:10 000, antimouse 1:5000 and antigoat 1:5000 (Jackson ImmunoResearch Laboratories, West Grove, PA). Immunoreactive bands were visualized using SuperSignal West Dura Chemiluminescent Substrate (Thermo Scientific, Burlington, ON). Relative band intensity was calculated using ImageLab software (Bio-Rad) and normalized to the quantified total protein on each respective membrane, as determined through Amido black staining (Aldridge, Podrebarac, Greenough, \& Weiler, 2008). 


\subsection{RNA extraction and real time-polymerase chain reaction}

Total RNA was extracted from homogenized 3-month heart samples using TRIzol reagent according to the manufacturer's instructions (Invitrogen). Two micrograms of RNA were reversed-transcribed to cDNA using the high-capacity cDNA Reverse Transcription Kit (Applied Biosystems, Beverly, MA). Primer sets for NADH:ubiquinone oxidoreductase core subunit V1 (ND1) (NM_001006972.1: forward 5'-CCGAGAACGCAACTCAGGTA-3'; reverse 5'-CCTAAGACACC ACCAGCATGT-3') and $\beta$-actin (NM_031144: forward $5^{\prime}$-CACAGC TGAGAGGGAAAT-3'; reverse $5^{\prime}$-TCAGCAATGCCTGGGTAC-3') were designed using the National Center for Biotechnology

TABLE 1 Postnatal outcome measures

\begin{tabular}{lcc} 
Outcome measure & Vehicle & Nicotine \\
\hline Litter size ( $n$ ) & $12.8 \pm 0.66$ & $13.8 \pm 0.87$ \\
Sex ratio (male/female) & $1.29 \pm 0.16$ & $1.50 \pm 0.21$ \\
\hline Birth weight (PND1) (g) & $6.66 \pm 0.17$ & $6.12 \pm 0.10^{* *}$ \\
\hline Body weight (PND21) (g) & $52.85 \pm 1.70$ & $51.93 \pm 1.40$ \\
\hline Body weight (3 months) (g) & $438.29 \pm 16.95$ & $459.67 \pm 10.14$
\end{tabular}

PND, postnatal day.

(A)
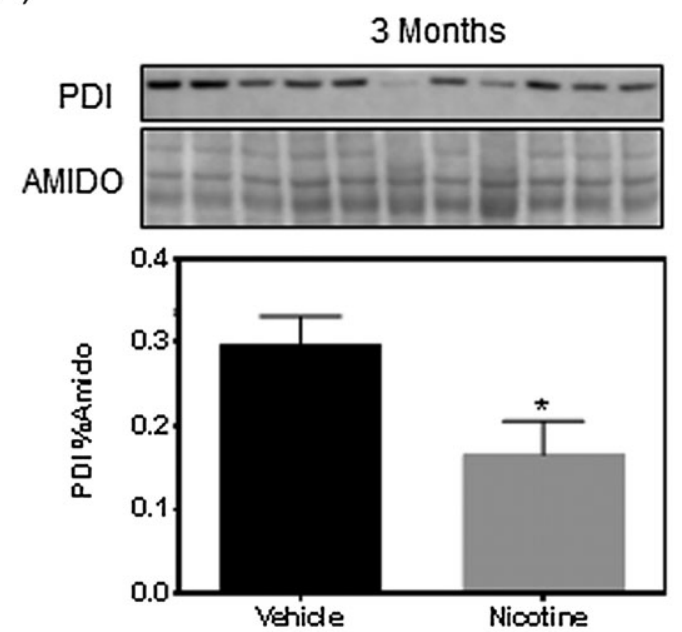

Treatment Groups

(C)

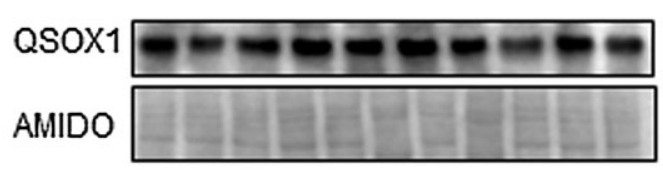

Information's primer designing tool and generated by Invitrogen Custom DNA Oligos. The relative transcript abundance was determined by quantitative real-time polymerase chain reaction using SensiFAST No-ROX SYBR Green Supermix (FroggaBio, North York, ON) and the Bio-Rad CFX384 Real Time System with the following cycling conditions: $95^{\circ} \mathrm{C}$ for 10 minutes, followed by 43 cycles of $95^{\circ} \mathrm{C}$ for $15 \mathrm{sec}-$ onds, $60^{\circ} \mathrm{C}$ for 30 seconds and $72^{\circ} \mathrm{C}$ for 30 seconds. Samples were assayed in triplicate and relative fold change was calculated using comparative cycle times $\left(C_{t}\right)$ method normalized to $\beta$-actin. $\Delta C_{t}$ values were standardized to the experimental sample with the lowest transcript abundance (highest $C_{\mathrm{t}}$ value). The relative abundance was calculated using the formula $2^{\Delta \Delta C t}$, where $\Delta \Delta C_{t}$ was the normalized value.

\section{4 | Statistical analysis}

All statistical analyses were performed using Graphpad Prism 6 software. Results from immunoblotting were presented as the mean of arbitrary values \pm SEM. Significant outliers were identified using the Grubbs' test. Data were tested for normality and equal variance. An unpaired Student's $t$-test was performed to examine results of immunoblotting data between control and MNE offspring. $P<0.05$ was deemed significant.

(B)
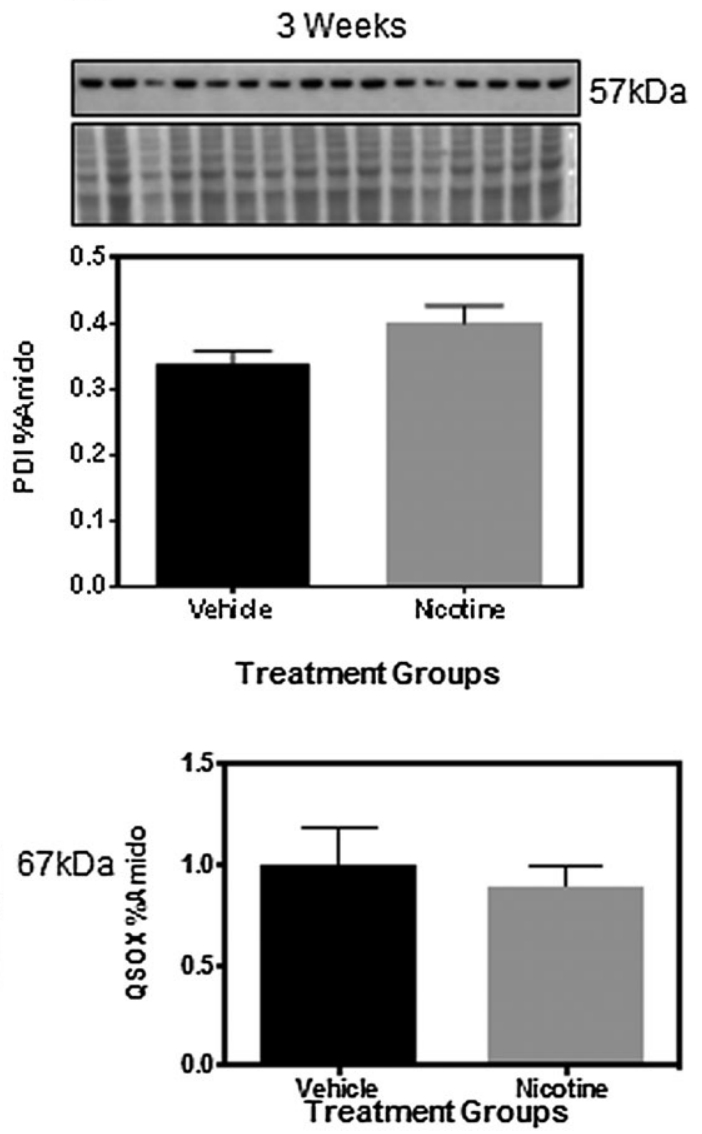

FIGURE 1 Perinatal nicotine exposure decreases PDI levels in the 3-month postnatal heart. Protein levels of targets of interest in 3-week and 3-month vehicle and perinatal nicotine-exposed cardiac tissue were determined via Western blot. From left to right, the first eight samples shown in the 3-week blots are vehicle controls, while the remaining samples are nicotine-exposed offspring. In the 3-month blots, the first five samples are vehicle controls, while the remaining samples are nicotine-exposed offspring. (A) PDI at 3 months. (B) PDI at 3 weeks. (C) QSOX1 at 3 months. All arbitrary values were expressed as means normalized to Amido black staining $(n=5-8$ per group). *significant difference $(P<0.05)$. PDI, protein disulfide isomerase 


\section{I RESULTS}

\section{1 | Maternal nicotine exposure decreases cardiac} protein disulfide isomerase protein levels at 3 months of age

Given previous work from our laboratory showed that nicotine exposure in vivo leads to decreased PDI levels in the rat placenta (Wong et al., 2015), we investigated if MNE altered cardiac PDI in the offspring. In this cohort of animals, MNE led to decreased birth weight $(p<0.01)$ followed by postnatal catch-up growth by three weeks of age compared to the vehicle controls (Table 1). Interestingly, PDI protein was significantly decreased in the hearts of MNE offspring at 3 months, but not at 3 weeks (Figure 1A,B). Similar to PDI, the quiescin-sulfhydryl oxidase (QSOX) family of flavoenzymes catalyzes the insertion of disulfide bonds into reduced unfolded proteins (Heckler, Rancy, Kodali, \& Thorpe, 2008). Given we have previously demonstrated that the ER oxidoreductase QSOX was decreased in the placenta due to MNE (Wong et al., 2015), we measured cardiac QSOX1 and found it was unaltered at 3 months between groups (Figure 1C).

\subsection{Maternal nicotine exposure does not lead to cardiac endoplasmic reticulum stress in maternal nicotine exposure offspring at 3 months of age}

As studies have demonstrated that a decrease in PDI can lead to augmented ER stress (Braakman \& Bulleid, 2011) and we have shown that nicotine leads to augmented ER stress in vivo and in vitro (Wong et al., 2015, 2016), we next investigated if markers of cardiac ER stress were evident in the 3-month MNE offspring in association with decreased PDI. Fetal and neonatal exposure to nicotine did not significantly alter protein expression of the key ER stress markers, Atf4, phospho-elF2a:elF2a, phospho-PERK, Grp78 or Grp94 at 3 months of age (Figure 2A-D).
(A)

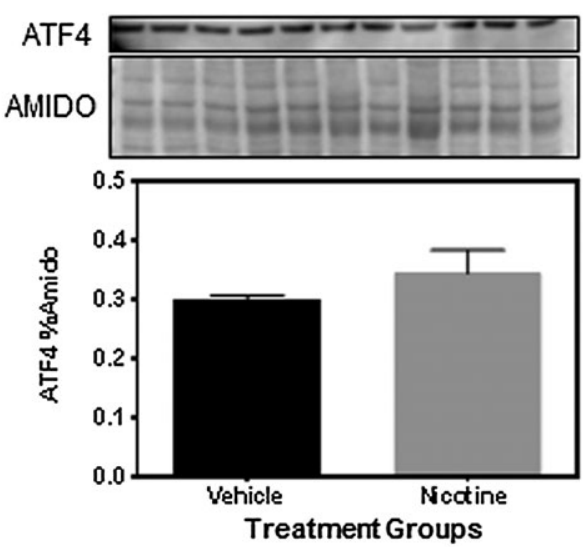

(C)
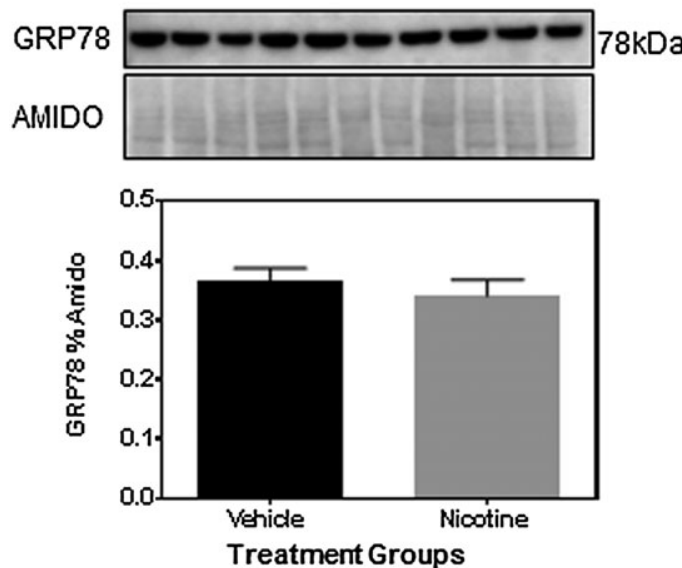

(B)
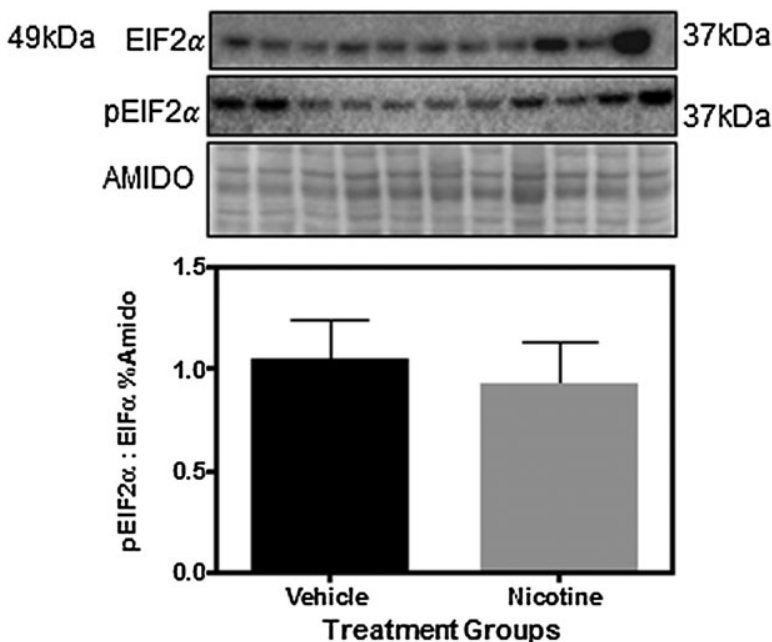

(D)
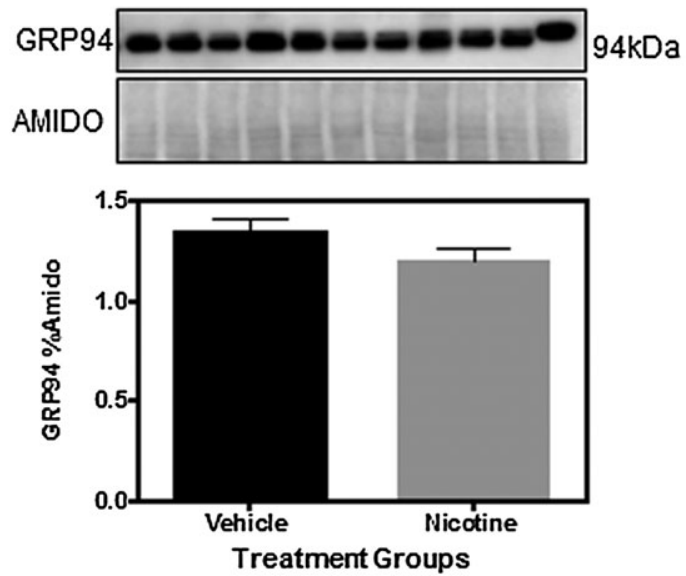

FIGURE 2 Perinatal nicotine exposure does not alter markers of endoplasmic reticulum stress in the 3-month postnatal heart. Protein levels of targets of interest in 3-month vehicle and perinatal nicotine-exposed cardiac tissue were determined via Western blot. From left to right, the first five samples shown in the 3-month blots are vehicle controls, while the remaining samples are nicotine-exposed offspring. (A) ATF4. (B) Ratio of phosphorylated elF2a: elF2 $a$. (C) GRP78. (D) GRP94. All arbitrary values were expressed as means normalized to Amido black staining ( $n=5-6$ per group) 


\subsection{Maternal nicotine exposure leads to decreased} protein levels of the antioxidant, superoxide

dismutase-2, in the hearts of 3-month-old rat offspring

Given the association between PDI levels and the antioxidant SOD-

1 expression and activity (Atkin et al., 2006; Toldo, Severino, et al., 2011b), we next investigated whether diminished cardiac PDI levels were linked to decreases in either SOD-1 or SOD-2 in these MNE offspring. At 3 months of age, MNE offspring displayed decreases in SOD-2 protein levels in the heart (Figure 3A), but this was not evident at 3 weeks (Figure 3B). Interestingly, the protein levels of cardiac SOD-1 were unaltered in these MNE offspring (Figure $3 \mathrm{C}$ ).
(A)

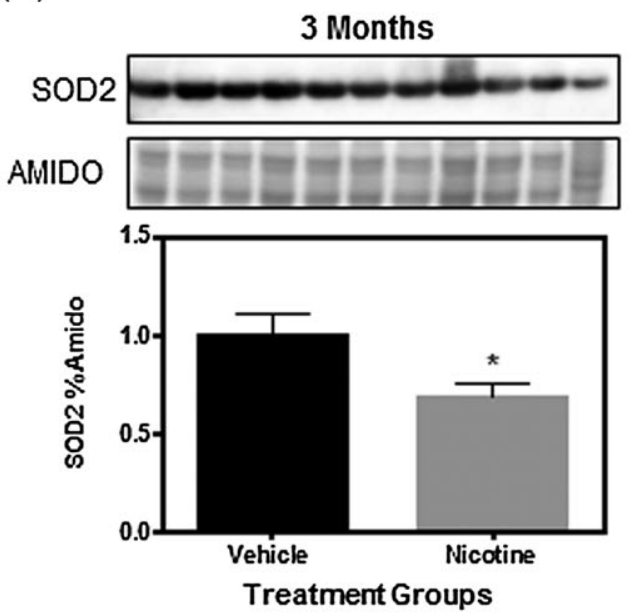

(C)

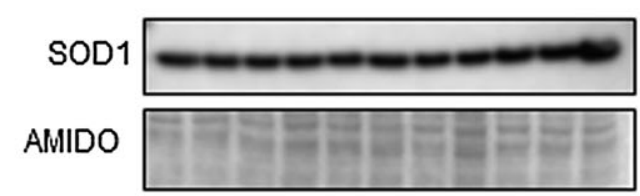

(D)

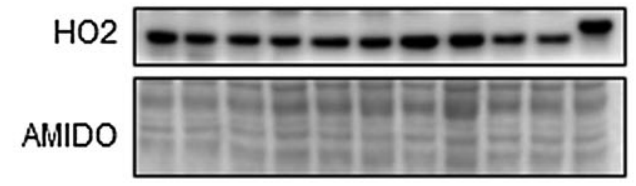

(E)

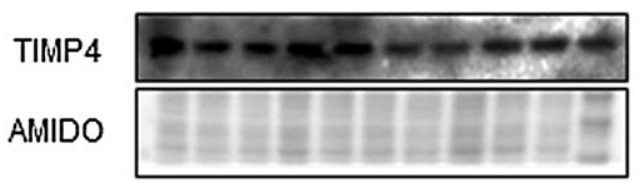

(B)

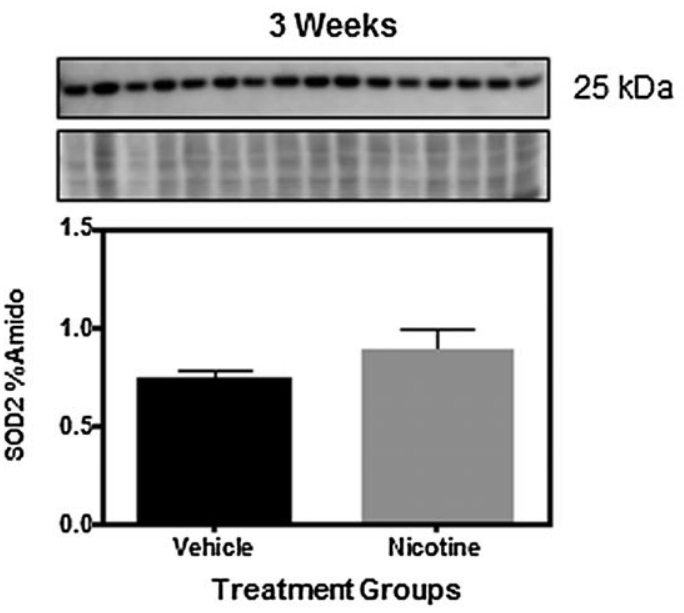

$23 \mathrm{kDa}$
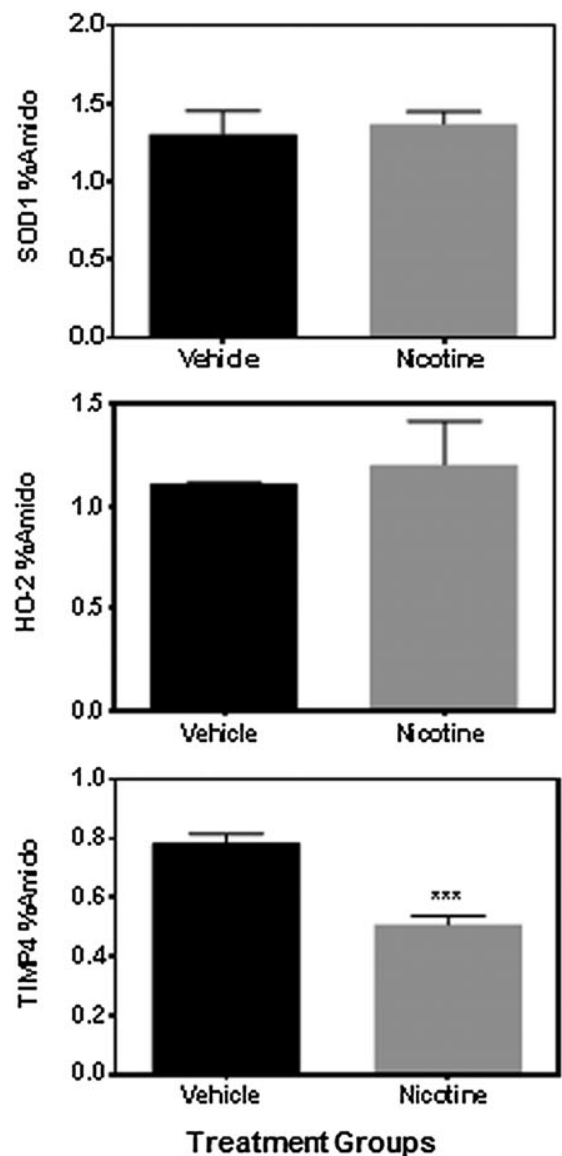

FIGURE 3 Perinatal nicotine exposure decreases the antioxidant SOD-2 concomitant with TIMP4 in the 3-month postnatal heart. Protein levels of targets of interest in 3-week and 3-month vehicle and perinatal nicotine-exposed cardiac tissue were determined via Western blot. From left to right, the first eight samples shown in the 3-week blots are vehicle controls, while the remaining samples are nicotine-exposed offspring. In the 3month blots, the first five samples are vehicle controls, while the remaining samples are nicotine-exposed offspring. (A) SOD-2 at 3 months. (B) SOD2 at 3 weeks. (C) SOD1 at 3 months. (D) HO-2 at 3 months. (E) TIMP4 at 3 months. All arbitrary values were expressed as means normalized to Amido black staining ( $n=5-8$ per group). *significant difference $(P<0.05)$. HO, heme oxygenase; SOD, superoxide dismutase; TIMP, tissue inhibitor of metalloproteinase 
Moreover, the levels of $\mathrm{HO}-2$, another antioxidant demonstrated to be decreased in heart failure (Ding, Li, \& Schultz, 2008), was unaltered in these MNE offspring at 3 months (Figure 3D). Since oxidative stress (e.g. reactive oxygen species [ROS]) leads to decreased cardiac TIMP4 (Donnini et al., 2008; Wang, Tang, Yan, \& Feng, 2016), a critical matrix metalloprotease for cardiac contractility and long-term health (Chaturvedi \& Tyagi, 2016; Felkin et al., 2006), we next measured its protein levels in our 3 month offspring. MNE offspring exhibited decreased cardiac TIMP4 levels at 3 months of age (Figure $3 \mathrm{E}$ ).

\subsection{Maternal nicotine exposure leads to impaired mitochondrial complex proteins in the hearts of 3- month-old rat offspring}

Since perinatal nicotine exposure results in postnatal pancreatic mitochondrial dysfunction and SOD-2 is exclusively localized in the mitochondria (compared to cytosolic SOD-1) (Bruin, Petre, Raha, et al., 2008b), we next investigated if mitochondrial number, mass and complex proteins might be impaired in the hearts of MNE offspring. At 3 months of age, perinatal nicotine exposure did not significantly alter the mRNA expression of ND-1 (Figure 4A), a marker of mitochondrial number (Ye et al., 2017). While there was a trend for decreased protein expression of citrate synthase, a marker of total mitochondrial mass, in nicotine-exposed offspring compared to vehicle controls, this was not significant (Figure 4B) (Figueiredo, Ferreira, Appell, \& Duarte, 2008). However, at 3 months of age, MNE offspring demonstrated lower protein levels of mitochondrial protein complex I, II, IV and V compared to vehicle-exposed offspring (Figure $5 \mathrm{~A} / \mathrm{C}$ ). At 3 weeks of age, MNE offspring did not exhibit any changes in mitochondrial complex proteins (I-V; Figure 5B/D).

\section{4 | DISCUSSION}

Animal studies now demonstrate the strong links between perinatal nicotine exposure and long-term cardiac dysfunction (Chou \& Chen, 2014; Fox et al., 2012; Gao et al., 2008; Lawrence et al., 2008; Wang et al., 2015; Xiao, Huang, Lawrence, Yang, \& Zhang, 2007; Xiao et al., 2016; Yu et al., 2016). While several of these cardiac defects have been attributed to increased fibrosis, little is known about the underlying cellular stresses involved in nicotine-induced cardiac disease in these offspring, particularly after the perinatal nicotine insult (Chou \& Chen, 2014; Yu et al., 2016). In the current study, we demonstrated that at 3 months of age, male offspring born to nicotine-exposed dams have decreased PDI levels in the hearts associated with decreased SOD-2 and mitochondrial complex expression. We chose this age given this was the earliest time point post-weaning when cardiac defects (i.e. higher heart rate, blood pressure) have been reported (Gao et al., 2008; Yu et al., 2016). Given cardiac PDI is critical for cardioprotection, the decrease in its levels in these nicotineexposed offspring reveals a potential mechanism to explain, in part, the observed impairments in recovery to ischemic injury (Lawrence et al., 2008; Toldo, Boccellino, et al., 2011a; Toldo, Severino, et al., 2011b; Xiao et al., 2016). Furthermore, the decreased cardiac PDI levels may also mediate the reduced levels of antioxidant SOD-2 observed in these nicotine-exposed hearts, particularly given the strong association between cardiac PDI and SOD (Toldo, Severino, et al., 2011b). In perinatal nicotine-exposed hearts, enhanced ROS production coupled with low levels of mitochondrial SOD-2 antioxidant likely mediates the observed impairment of mitochondrial complex proteins (I, II, IV and V) expression in the heart (Xiao et al., 2016). As previously demonstrated, SOD-2 deficiency in mice leads to decreased mitochondrial complex I and/or II activity in the heart (Li et al., 1995; Melov et al., 1999). Since decreased antioxidant
(A)

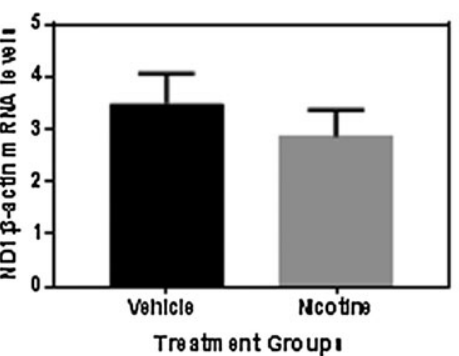

(B)

cs

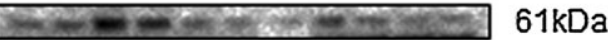

AMIDO
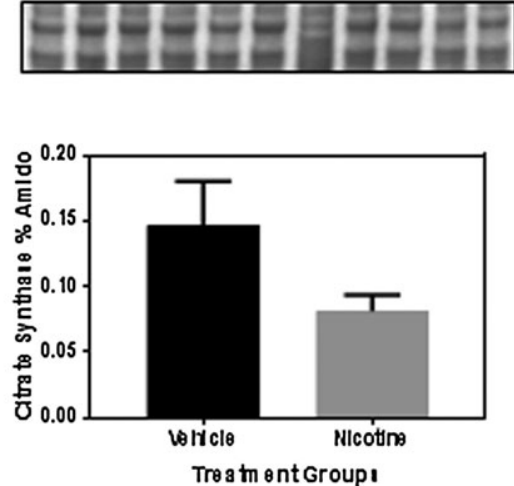

FIGURE 4 Perinatal nicotine exposure does not alter mitochondrial number and mass in the 3-month postnatal heart. mRNA and protein levels of targets of interest in 3-month vehicle and perinatal nicotine-exposed cardiac tissue were determined via quantitated real-time polymerase chain reaction and Western blot, respectively. (A) NADH:Ubiquinone oxidoreductase core subunit V1 (ND1) and (B) CS at 3 months. From left to right, the first five samples shown in the 3-month blot are vehicle controls, while the remaining samples are nicotine-exposed offspring. All arbitrary values were expressed as means normalized to (a) $\beta$-actin and to (B) Amido black staining ( $n=5-6$ per group). ${ }^{*}$ significant difference $(P<0.05)$. CS, citrate synthase 
(A)

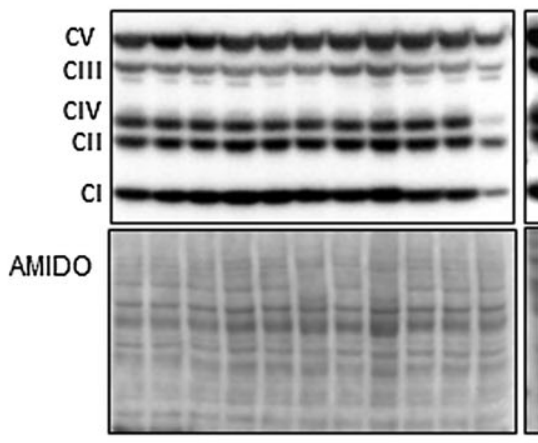

(C)
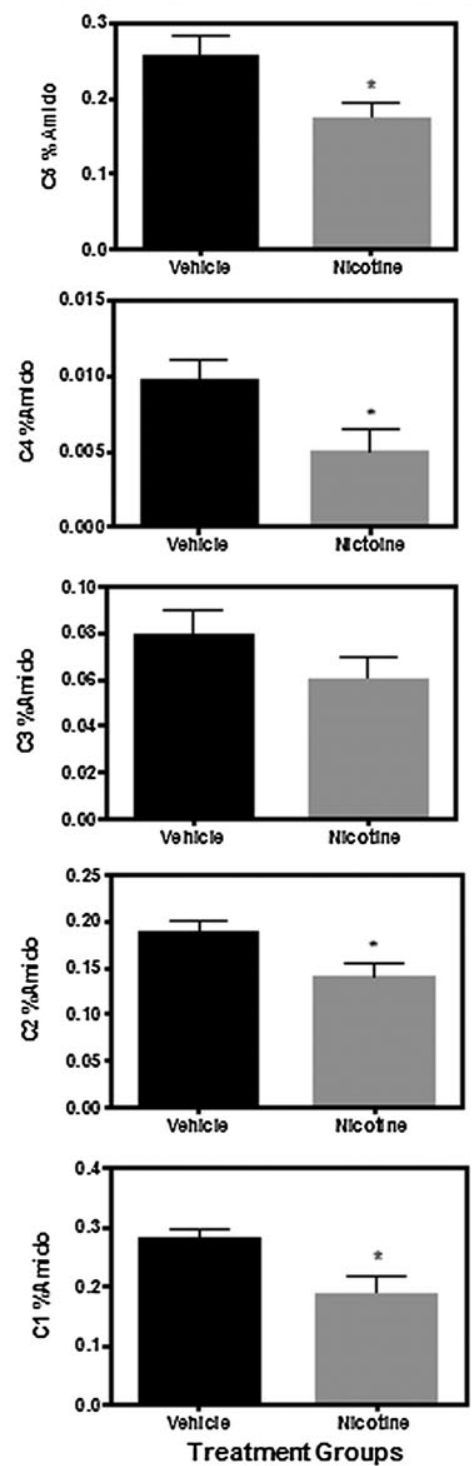

(B)
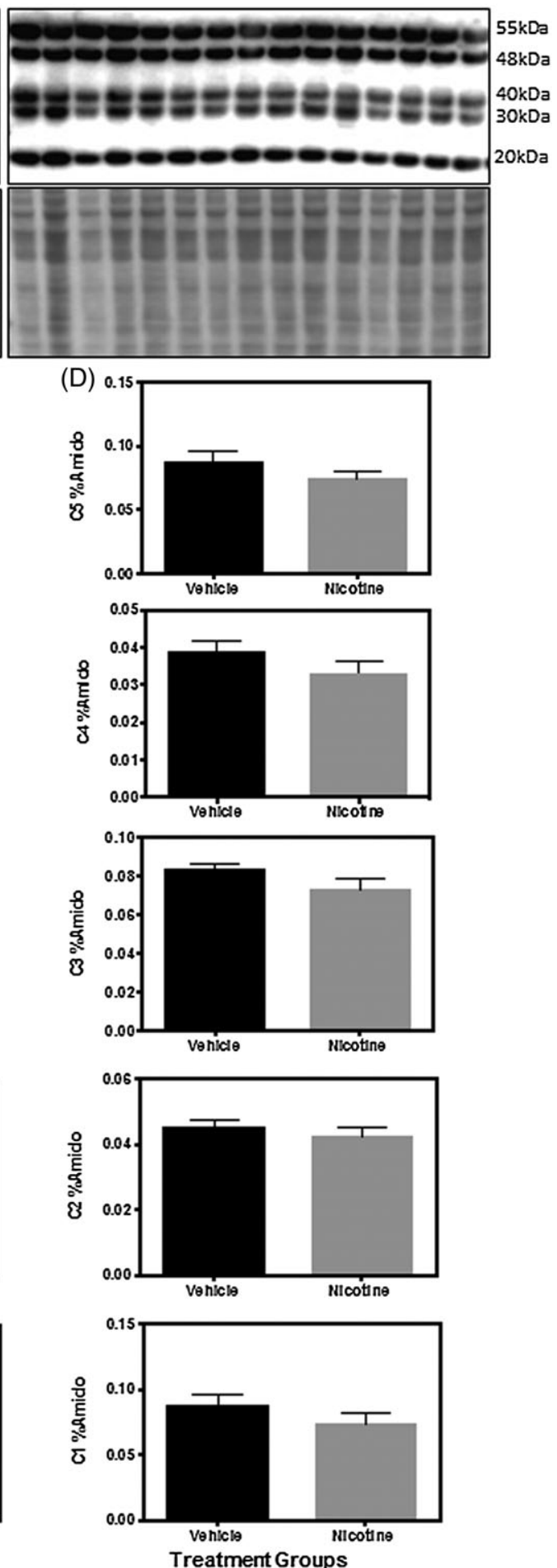

FIGURE 5 Perinatal nicotine exposure decreases mitochondrial complexes I, II, IV and V in the 3-month postnatal heart. Protein levels of targets of interest in 3-week and 3-month vehicle and perinatal nicotine-exposed cardiac tissue were determined via Western blot using the OXPHOS antibody. From left to right, the first eight samples in the 3-week blots are vehicle controls, while the remaining samples are nicotine-exposed offspring. In the 3-month blots, the first five samples are vehicle controls, while the remaining samples are nicotine-exposed offspring. (A) mitochondrial complexes I-V at 3 months and (B) at 3 weeks. All arbitrary values were expressed as means normalized to Amido black staining ( $n=5-8$ per group). *significant difference $(P<0.05)$

enzyme expression and reductions in mitochondrial ETC protein expression have been associated with increased cellular stress (Tatarkova et al., 2016; Vidimar et al., 2016; Wiegman et al., 2015), it is not surprising that these offspring exhibit lower levels of cardiac TIMP4. TIMP4 is a matrix metalloproteinase inhibitor that is linked to long-term cardiac health and both its expression and activity is impaired by oxidative stress (Chaturvedi \& Tyagi, 2016; Donnini et al., 2008; Felkin et al., 2006; Wang et al., 2016). Moreover, increased oxidative stress and reduced mitochondrial complex proteins in nicotine-exposed offspring has been previously implicated in 
cardiac fibrosis and long-term heart failure (Aragno et al., 2006; Kuroda et al., 2010).

In placenta, we have shown that nicotine exposure decreased PDI expression and increased ER stress (Wong et al., 2015). However, in the heart although we saw diminished PDI levels, it was not associated with activation of the core components of the ER stress pathway suggesting that at least in this tissue PDI and ER stress may not be coupled. Indeed, in our model decreased PDI appears to be associated with redox imbalance, likely stemming from impaired mitochondrial function (Ferreiro et al., 2012). The notion that PDI and oxidative stress are intimately linked is based on several studies that suggest that $\mathrm{PDI}$ is tightly associated with $\mathrm{NAD}(\mathrm{P}) \mathrm{H}$ oxidase and acts as a nove redox-sensitive regulatory protein (Janiszewski et al., 2005; Laurindo, Fernandes, Amanso, Lopes, \& Santos, 2008).

Given the links between PDI and the antioxidant SOD, we then determined if these nicotine-exposed offspring exhibit changes in antioxidant expression. SOD-1 is mainly localized in the cytosol, while SOD-2 is exclusively expressed in the mitochondria. While previous studies have more closely linked PDI to SOD-1, our studies identified that SOD-2 levels were lower at 3-month offspring due to perinatal nicotine exposure, but not SOD-1 (Atkin et al., 2006; Toldo, Severino, et al., 2011b). The decrease in SOD-2 early in life may help elucidate, in part, why in utero nicotine-exposed offspring exhibit increases in cardiac ROS production (Xiao et al., 2016). Moreover, higher ROS levels have been demonstrated to inhibit cardiac TIMP4 providing a conceivable mechanism for the decreased TIMP4 levels we observed in these nicotine-exposed offspring (Donnini et al., 2008; Wang et al., 2016). The diminished levels of SOD-2 versus cytosolic SOD-1 or HO-2 might suggest that oxidative stress is occurring mainly in the mitochondria.

Fetal and neonatal exposure to nicotine has been previously demonstrated to cause oxidative stress and impaired mitochondrial function in other tissues (Bruin et al., 2008a, b). It appears that a similar effect occurs in the heart as the expression of the mitochondrialspecific antioxidant enzyme SOD-2 is decreased in association with decreases in the mitochondrial ETC proteins, independent of altered mitochondrial mass and number. However, as the majority of these observations are made in tissues well after the critical period of nicotine exposure, little is understood regarding the mechanism of these mitochondrial defects. Given the strong links between PDI and SOD, and that both PDI and SOD-2 can be localized to mitochondria, we speculate the loss of PDI at 3 months may indirectly govern the mitochondrial dysfunction observed (Atkin et al., 2006; Rigobello, Donella-Deana, Cesaro, \& Bindoli, 2001; Toldo, Severino, et al., 2011b).

While there is a disconnect between the direct effects of nicotine at 3 weeks compared to the long-term effects on PDI, SOD-2 and mitochondrial complexes, one conceivable explanation for this may be due to the fact that perinatal nicotine exposure leads to postnatal catch-up growth in these offspring by 3 months of age (Yu et al., 2016). This is important given that in other rat models of IUGR accompanied with postnatal catch-up growth, increases in oxidative stress (e. g. higher ROS levels and lower antioxidant enzymes) were apparent in the heart (Tarry-Adkins et al., 2013). Moreover, postnatal catch-up growth is also associated with decreases in hepatic mitochondrial complex (I-IV) activity and lower mitochondrial citrate activity (Zheng et al.,
2011). Xiao et al. recently demonstrated that the use of the ROS inhibitor $\mathrm{N}$-acetyl-cysteine during pregnancy could ameliorate the adverse effects of nicotine on both long-term oxidative stress and impaired recovery to ischemia (Xiao et al., 2016). However, appreciation of the indirect effects of nicotine on long-term cardiac dysfunction in postnatal life is warranted in a clinical setting given interventions in human pregnancy may not be safe for the mother or fetus. More importantly, postnatal interventions may be a potential therapeutic strategy for children, who without choice, were exposed to smoking and/or nicotine replacement therapy. There may be promise in postnatal interventions, given studies which demonstrate that the use of antioxidants (i.e. coenzyme Q10, resveratrol) during the period postnatal catch-up growth can prevent long-term oxidative stress, mitochondrial dysfunction and premature aging in hepatic and cardiac tissue of IUGR offspring (Tarry-Adkins et al., 2013; Zheng et al., 2011). Regardless of the timing of intervention, the outcomes of this study would suggest that pharmaceuticals boosting PDI expression and/or activity might be efficacious for nicotine-exposed offspring, particularly given its well-established cardio-protective role and association with cytosolic and mitochondrial antioxidants.

\section{CONFLICT OF INTEREST}

The authors did not report any conflict of interest.

\section{ACKNOWLEDGEMENTS}

This work was supported by the Canadian Institutes for Health Research (MOP86474 to $\mathrm{ACH}$ and MOP 111011 to $\mathrm{DBH}$ ) and a research grant from the Women's Development Council to $\mathrm{DBH}$. NGB is a recipient of both a Molly Towell Perinatal Research Foundation Fellowship and a Whaley Postdoctoral Fellowship. ML is a recipient of the Dean's Undergraduate Research Opportunities Program award.

\section{REFERENCES}

Aldridge, G. M., Podrebarac, D. M., Greenough, W. T., \& Weiler, I. J. (2008). The use of total protein stains as loading controls: An alternative to high-abundance single-protein controls in semi-quantitative immunoblotting. Journal of Neuroscience Methods, 172(2), 250-254. https:// doi.org/10.1016/j.jneumeth.2008.05.003

Aragno, M., Mastrocola, R., Medana, C., Catalano, M. G., Vercellinatto, I., Danni, O., \& Boccuzzi, G. (2006). Oxidative stress-dependent impairment of cardiac-specific transcription factors in experimental diabetes. Endocrinology, 147(12), 5967-5974. https://doi.org/10.1210/ en.2006-0728

Atkin, J. D., Farg, M. A., Turner, B. J., Tomas, D., Lysaght, J. A., Nunan, J., ... Horne, M. K. (2006). Induction of the unfolded protein response in familial amyotrophic lateral sclerosis and association of protein-disulfide isomerase with superoxide dismutase 1. Journal of Biological Chemistry, 281(40), 30152-30165. https://doi.org/10.1074/jbc. M603393200

Braakman, I., \& Bulleid, N. J. (2011). Protein folding and modification in the mammalian endoplasmic reticulum. Annual Review of Biochemistry, 80, 71-99. https://doi.org/10.1146/annurev-biochem-062209-093836

Bruin, J. E., Petre, M. A., Lehman, M. A., Raha, S., Gerstein, H. C., Morrison, K. M., \& Holloway, A. C. (2008a). Maternal nicotine exposure increases oxidative stress in the offspring. Free Radical Biology \& Medicine, 44(11), 1919-1925. https://doi.org/10.1016/j.freeradbiomed.2008.02.010 
Bruin, J. E., Petre, M. A., Raha, S., Morrison, K. M., Gerstein, H. C., \& Holloway, A. C. (2008b). Fetal and neonatal nicotine exposure in Wistar rats causes progressive pancreatic mitochondrial damage and beta cell dysfunction. PloS One, 3(10). e3371. https://doi.org/10.1371/journal. pone.0003371

Chaturvedi, P., \& Tyagi, S. C. (2016). Epigenetic silencing of TIMP4 in heart failure. Journal of Cellular and Molecular Medicine, 20(11), 2089-2101. https://doi.org/10.1111/jcmm.12901

Chou, H. C., \& Chen, C. M. (2014). Maternal nicotine exposure during gestation and lactation induces cardiac remodeling in rat offspring. Reproductive Toxicology (Elmsford, N.Y.), 50, 4-10. https://doi.org/ 10.1016/j.reprotox.2014.09.013

Cui, Y., Shooshtari, S., Forget, E. L., Clara, I., \& Cheung, K. F. (2014). Smoking during pregnancy: Findings from the 2009-2010 Canadian community health survey. PloS One, 9. e84640. https://doi.org/ 10.1371/journal.pone.0084640

Dasgupta, C., Xiao, D., Xu, Z., Yang, S., \& Zhang, L. (2012). Developmental nicotine exposure results in programming of alveolar simplification and interstitial pulmonary fibrosis in adult male rats. Reproductive Toxicology (Elmsford, N.Y.), 34(3), 370-377. https://doi.org/10.1016/j. reprotox.2012.05.100

De Long, N. E., Barra, N. G., Hardy, D. B., \& Holloway, A. C. (2014). Is it safe to use smoking cessation therapeutics during pregnancy? Expert Opinion on Drug Safety, 13(12), 1721-1731. https://doi.org/10.1517/ 14740338.2014.973846

Ding, Y., Li, Y.-L., \& Schultz, H. D. (2008). Downregulation of carbon monoxide as well as nitric oxide contributes to peripheral chemoreflex hypersensitivity in heart failure rabbits. Journal of Applied Physiology (Bethesda, Md.: 1985), 105, 14-23. https://doi.org/10.1152/ japplphysiol.01345.2007

Donnini, S., Monti, M., Roncone, R., Morbidelli, L., Rocchigiani, M., Oliviero, S., ... Ziche, M. (2008). Peroxynitrite inactivates human-tissue inhibitor of metalloproteinase-4. FEBS Letters, 582(7), 1135-1140. https://doi. org/10.1016/j.febslet.2008.02.080

Felkin, L. E., Birks, E. J., George, R., Wong, S., Khaghani, A., Yacoub, M. H., \& Barton, P. J. R. (2006). A quantitative gene expression profile of matrix metalloproteinases (MMPS) and their inhibitors (TIMPS) in the myocardium of patients with deteriorating heart failure requiring left ventricular assist device support. Journal of Heart and Lung Transplantation, 25(12), 1413-1419. https://doi.org/10.1016/j. healun.2006.09.006

Ferreiro, E., Baldeiras, I., Ferreira, I. L., Costa, R. O., Rego, A. C., Pereira, C. F., \& Oliveira, C. R. (2012). Mitochondrial- and endoplasmic reticulumassociated oxidative stress in Alzheimer's disease: From pathogenesis to biomarkers. International Journal of Cell Biology, 2012, 735206. https://doi.org/10.1155/2012/735206

Figueiredo, P. A., Ferreira, R. M., Appell, H. J., \& Duarte, J. A. (2008). Ageinduced morphological, biochemical, and functional alterations in isolated mitochondria from murine skeletal muscle. Journals of Gerontology. Series A, Biological Sciences and Medical Sciences, 63(4), 350-359.

Fox, K. A., Longo, M., Tamayo, E., Gamble, P., Makhlouf, M., Mateus, J. F., \& Saade, G. R. (2012). Sex-specific effects of nicotine exposure on developmental programming of blood pressure and vascular reactivity in the C57BI/6J mouse. American Journal of Obstetrics and Gynecology, 207(3). 208.e1-9. https://doi.org/10.1016/j.ajog.2012.06.021

Gao, Y. J., Holloway, A. C., Su, L. Y., Takemori, K., Lu, C., \& Lee, R. M. (2008). Effects of fetal and neonatal exposure to nicotine on blood pressure and perivascular adipose tissue function in adult life. European Journal of Pharmacology, 590(1-3), 264-268. https://doi.org/10.1016/j. ejphar.2008.05.044

Gao, Y. J., Holloway, A. C., Zeng, Z. H., Lim, G. E., Petrik, J. J., Foster, W. G., \& Lee, R. M. (2005). Prenatal exposure to nicotine causes postnatal obesity and altered perivascular adipose tissue function. Obesity Research, 13(4), 687-692. https://doi.org/10.1038/oby.2005.77

Heckler, E. J., Rancy, P. C., Kodali, V. K., \& Thorpe, C. (2008). Generating disulfides with the quiescin-sulfhydryl oxidases. Biochimica et
Biophysica Acta, 1783(4), 567-577. https://doi.org/10.1016/j. bbamcr.2007.10.002

Holloway, A. C., Kellenberger, L. D., \& Petrik, J. J. (2006). Fetal and neonatal exposure to nicotine disrupts ovarian function and fertility in adult female rats. Endocrine, 30(2), 213-216. https://doi.org/10.1385/ ENDO:30:2:213

Holloway, A. C., Salomon, A., Soares, M. J., Garnier, V., Raha, S., Sergent, F., ... Alfaidy, N. (2014). Characterization of the adverse effects of nicotine on placental development: In vivo and in vitro studies. American Journal of physiology.Endocrinology and. Metabolism, 306(4), E443-E456. https://doi.org/10.1152/ajpendo.00478.2013

Janiszewski, M., Lopes, L. R., Carmo, A. O., Pedro, M. A., Brandes, R. P., Santos, C. X. C., \& Laurindo, F. R. M. (2005). Regulation of NAD(P)H oxidase by associated protein disulfide isomerase in vascular smooth muscle cells. Journal of Biological Chemistry, 280(49), 40813-40819. https://doi.org/10.1074/jbc.M509255200

Kuroda, J., Ago, T., Matsushima, S., Zhai, P., Schneider, M. D., \& Sadoshima, J. (2010). NADPH oxidase 4 (Nox4) is a major source of oxidative stress in the failing heart. Proceedings of the National Academy of Sciences of the United States of America, 107(35), 15565-15570. https://doi.org/ 10.1073/pnas.1002178107

Laurindo, F. R. M., Fernandes, D. C., Amanso, A. M., Lopes, L. R., \& Santos, C. X. C. (2008). Novel role of protein disulfide isomerase in the regulation of NADPH oxidase activity: Pathophysiological implications in vascular diseases. Antioxidants \& Redox Signaling, 10(6), 1101-1113. https://doi.org/10.1089/ars.2007.2011

Lawrence, J., Xiao, D., Xue, Q., Rejali, M., Yang, S., \& Zhang, L. (2008). Prenatal nicotine exposure increases heart susceptibility to ischemia/ reperfusion injury in adult offspring. Journal of Pharmacology and Experimental Therapeutics, 324, 331-341. https://doi.org/10.1124/ jpet.107.132175

Li, Y., Huang, T. T., Carlson, E. J., Melov, S., Ursell, P. C., Olson, J. L., ... Epstein, C. J. (1995). Dilated cardiomyopathy and neonatal lethality in mutant mice lacking manganese superoxide dismutase. Nature Genetics, 11(4), 376-381. https://doi.org/10.1038/ng1295-376

Ma, N., Nicholson, C. J., Wong, M., Holloway, A. C., \& Hardy, D. B. (2014). Fetal and neonatal exposure to nicotine leads to augmented hepatic and circulating triglycerides in adult male offspring due to increased expression of fatty acid synthase. Toxicology and Applied Pharmacology, 275, 1-11. https://doi.org/10.1016/j.taap.2013.12.010

Melov, S., Coskun, P., Patel, M., Tuinstra, R., Cottrell, B., Jun, A. S., ... Wallace, D. C. (1999). Mitochondrial disease in superoxide dismutase 2 mutant mice. Proceedings of the National Academy of Sciences of the United States of America, 96(3), 846-851.

Pauly, J. R., \& Slotkin, T. A. (2008). Maternal tobacco smoking, nicotine replacement and neurobehavioural development. Acta Paediatrica (Oslo, Norway: 1992), 97(10), 1331-1337. https://doi.org/10.1111/j.16512227.2008.00852.x

Rigobello, M. P., Donella-Deana, A., Cesaro, L., \& Bindoli, A. (2001). Distribution of protein disulphide isomerase in rat liver mitochondria. Biochemical Journal, 356(Pt 2), 567-570.

Rush, J. W. E., Green, H. J., Maclean, D. A., \& Code, L. M. (2005). Oxidative stress and nitric oxide synthase in skeletal muscles of rats with postinfarction, compensated chronic heart failure. Acta Physiologica Scandinavica, 185(3), 211-218. https://doi.org/10.1111/j.1365201X.2005.01479.x

Tarry-Adkins, J. L., Martin-Gronert, M. S., Fernandez-Twinn, D. S., Hargreaves, I., Alfaradhi, M. Z., Land, J. M., ... Ozanne, S. E. (2013). Poor maternal nutrition followed by accelerated postnatal growth leads to alterations in DNA damage and repair, oxidative and nitrosative stress, and oxidative defense capacity in rat heart. FASEB Journal, 27, 379390. https://doi.org/10.1096/fj.12-218685

Tatarkova, Z., Kovalska, M., Timkova, V., Racay, P., Lehotsky, J., \& Kaplan, P. (2016). The effect of aging on mitochondrial complex I and the extent of oxidative stress in the rat brain cortex. Neurochemical Research, 41(8), 2160-2172. https://doi.org/10.1007/s11064-016-1931-z 
Toldo, S., Boccellino, M., Rinaldi, B., Seropian, I. M., Mezzaroma, E., Severino, A., ... Baldi, A. (2011a). Altered oxido-reductive state in the diabetic heart: Loss of cardioprotection due to protein disulfide isomerase. Molecular Medicine (Cambridge, Mass.), 17(9-10), 1012-1021. https://doi.org/10.2119/molmed.2011.00100

Toldo, S., Severino, A., Abbate, A., \& Baldi, A. (2011b). The role of PDI as a survival factor in cardiomyocyte ischemia. Methods in Enzymology, 489, 47-65. https://doi.org/10.1016/B978-0-12-385116-1.00003-0

Vidimar, V., Gius, D., Chakravarti, D., Bulun, S. E., Wei, J. J., \& Kim, J. J. (2016). Dysfunctional MnSOD leads to redox dysregulation and activation of prosurvival AKT signaling in uterine leiomyomas. Science Advances, 2(11). e1601132. https://doi.org/10.1126/ sciadv.1601132

Wang, Z., Ouyang, Q., Huang, Z., Lin, L., Yu, E., \& Ferrari, M. W. (2015). Prenatal nicotine exposure induces gender-associated left ventriculararterial uncoupling in adult offspring. Molecular Medicine Reports, 12, 410-418. https://doi.org/10.3892/mmr.2015.3364

Wang, G., Tang, C., Yan, G., \& Feng, B. (2016). Gene expression profiling of $\mathrm{H} 9 \mathrm{c} 2$ cells subjected to $\mathrm{H}_{2} \mathrm{O}_{2}$-induced apoptosis with/without AFHF001. Biological \& Pharmaceutical Bulletin, 39(2), 207-214. https:// doi.org/10.1248/bpb.b15-00601

Wiegman, C. H., Michaeloudes, C., Haji, G., Narang, P., Clarke, C. J., Russell, K. E., ... COPDMAP, COPDMAP (2015). Oxidative stress-induced mitochondrial dysfunction drives inflammation and airway smooth muscle remodeling in patients with chronic obstructive pulmonary disease. Journal of Allergy and Clinical Immunology, 136(3), 769-780. https:// doi.org/10.1016/j.jaci.2015.01.046

Wong, M. K., Holloway, A. C., \& Hardy, D. B. (2016). Nicotine directly induces endoplasmic reticulum stress response in rat placental trophoblast giant cells. Toxicological Sciences, 151, 23-34. https://doi.org/ 10.1093/toxsci/kfw019

Wong, M. K., Nicholson, C. J., Holloway, A. C., \& Hardy, D. B. (2015). Maternal nicotine exposure leads to impaired disulfide bond formation and augmented endoplasmic reticulum stress in the rat placenta. PloS One, 10(3). e0122295. https://doi.org/10.1371/journal.pone.0122295

Xiao, D., Huang, X., Lawrence, J., Yang, S., \& Zhang, L. (2007). Fetal and neonatal nicotine exposure differentially regulates vascular contractility in adult male and female offspring. Journal of Pharmacology and
Experimental Therapeutics, 320(2), 654-661. https://doi.org/10.1124/ jpet.106.113332

Xiao, D., Wang, L., Huang, X., Li, Y., Dasgupta, C., \& Zhang, L. (2016). Protective effect of antenatal antioxidant on nicotine-induced heart ischemia-sensitive phenotype in rat offspring. PloS One, 11(2). e0150557. https://doi.org/10.1371/journal.pone.0150557

Xiao, D., Xu, Z., Huang, X., Longo, L. D., Yang, S., \& Zhang, L. (2008). Prenatal gender-related nicotine exposure increases blood pressure response to angiotensin II in adult offspring. Hypertension, 51(4), 1239-1247. https://doi.org/10.1161/HYPERTENSIONAHA.107.106203

Ye, W., Tang, X., Liu, C., Wen, C., Li, W., \& Lyu, J. (2017). Accurate quantitation of circulating cell-free mitochondrial DNA in plasma by droplet digital PCR. Analytical and Bioanalytical Chemistry. https://doi.org/ 10.1007/s00216-017-0217-x

Yu, S. J., Yoon, J. H., Yang, J. I., Cho, E. J., Kwak, M. S., Jang, E. S., ... Kim, C. Y. (2012). Enhancement of hexokinase II inhibitor-induced apoptosis in hepatocellular carcinoma cells via augmenting ER stress and anti-angiogenesis by protein disulfide isomerase inhibition. Journal of Bioenergetics and Biomembranes, 44, 101-115. https://doi.org/ 10.1007/s10863-012-9416-5

Yu, F., Zheng, A., Qian, J., Li, Y., Wu, L., Yang, J., \& Gao, X. (2016). Prenatal nicotine exposure results in the myocardial fibrosis in the adult male offspring rats. Experimental and Toxicologic Pathology, 68(8), 445-450. https://doi.org/10.1016/j.etp.2016.07.002

Zheng, J., Chen, L. L., Zhang, H. H., Hu, X., Kong, W., \& Hu, D. (2011). Resveratrol improves insulin resistance of catch-up growth by increasing mitochondrial complexes and antioxidant function in skeletal muscle. Metabolism: Clinical and Experimental, 61(7), 954-965. https://doi.org/ 10.1016/j.metabol.2011.11.005

How to cite this article: Barra NG, Lisyansky M, Vanduzer TA, Raha S, Holloway AC, Hardy DB. Maternal nicotine exposure leads to decreased cardiac protein disulfide isomerase and impaired mitochondrial function in male rat offspring. J Appl Toxicol. 2017;1-10. https://doi.org/10.1002/jat.3503 